\title{
Sistema Automatizado de Estacionamiento para Patentes Argentinas
}

\author{
Automated LPR Parking System for Argentinian Plates \\ Juan Pablo Valeo ${ }^{\dagger}$, Sebastián Gregoracci ${ }^{\dagger}$, Leticia M. Seijas ${ }^{\dagger * 1}$, Juan A. Etcheverry ${ }^{\dagger 2}$
${ }^{\dagger}$ Instituto de Investigaciones Científicas y Tecnológicas en Electrónica (ICYTE), Universidad Nacional de Mar del Plata \\ Juan B. Justo 4302, Mar del Plata, Argentina

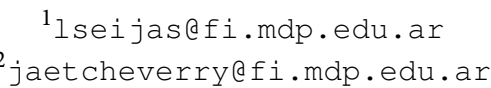 \\ * Departamento de Ingeniería Informática, Universidad Nacional de Mar del Plata \\ Juan B. Justo 4302, Mar del Plata, Argentina
}

Recibido: 16/10/20; Aceptado: 27/11/20

\begin{abstract}
The global market for smart parking solutions is booming. Nowadays, this market is highly competitive due mainly to the increase in car ownership and the number of vehicles circulating the roads. However, there is a technological gap between Argentina and other countries. This work presents the Automated LPR Parking System for Argentinian Plates SAE (in Spanish, Sistema Automatizado de Estacionamiento) designed and developed in its prototype version for a private company, to control and manage the entry, stay and exit of vehicles in parking lots. The system is composed by two main modules: the automatic license plate recognition system and the Principal System Board. This board allows the connection of the central unit with peripherals such as magnetic field sensors, access barriers and infrared barriers. In addition, the system manages a database that stores the necessary information for the parking management. Registered users that pay monthly and ocasinal users are considered. Furthermore, the system implements a mechanism for vehicle size detection to determine the value of the parking fees. SAE handles both hardware and system failures and exceptional situations. In addition, the entry and exit of vehicles are handled simultaneously.
\end{abstract}

Keywords: Parking Automation; ALPR; Printed Circuit Board.

Resumen- El mercado global de soluciones inteligentes para estacionamiento está en auge siendo altamente competitivo debido principalmente al crecimiento del parque automotor. En Argentina aún prevalece la operatoria manual. Este trabajo presenta el Sistema Automatizado de Estacionamiento (SAE), diseñado y desarrollado en su versión prototipo para una empresa privada, para controlar y gestionar el ingreso, egreso y estadía de vehículos en playas de estacionamiento. El sistema cuenta con un módulo de reconocimiento de patentes vigentes en Argentina, la placa principal que permite conectar la unidad central del sistema con los periféricos como detectores magnéticos, barreras de acceso y barreras infrarrojas, y una base de datos que almacena información necesaria para la gestión del estacionamiento. Se contemplan usuarios abonados y no abonados y tarifas para distintos tipos de vehículo, para lo cual se implementa un sistema para la detección de tamaño. EI SAE maneja casos de fallo tanto de hardware como del sistema y situaciones de excepción. Se contemplan además vías de ingreso y egreso con entrada y salida de vehículos en simultáneo.

Palabras clave: Automatización de estacionamientos; ALPR; placa circuital impresa.

\section{INTRODUCCIÓN}

En los últimos años el parque automotor de la República Argentina creció a grandes pasos. Sólo entre 2011 y 2017 aumentó en un $30 \%$ [1]. Cada vez hay más autos circulando por las calles y los espacios disponibles para estacionar sobre las mismas se están agotando. A pesar de ello, los autos se siguen fabricando y patentando y es por todo esto que las playas de estacionamiento cobran fuerza. En el plano internacional también se registra el crecimiento del parque automotor siendo la tendencia actual el automatizar los espacios de estacionamiento, ya sea en aeropuertos, cocheras, centros de compras, edificios privados, entre otros [2]. De esta manera, el mercado global de soluciones inteligentes para estacionamiento está en auge siendo altamente competitivo.

Este trabajo presenta el Sistema Automatizado de Estacionamiento (SAE) que controla y gestiona el ingreso, egreso y estadía de vehículos en playas de estacionamiento. El proyecto es impulsado por una empresa de la ciudad de Mar del Plata que relevó la necesidad de automatización de las playas de estacionamiento a nivel local. La falta de automatización y el uso de sistemas muy elementales genera una serie de vulnerabilidades al momento de asegurar el control total de las transacciones. A futuro, el sistema propuesto podría aplicarse a cocheras de edificios comerciales y privados, control de ingreso y egreso a barrios cerrados, entre otros.

La Sección II presenta aspectos del diseño del sistema y su funcionamiento. La Sección III describe la construcción del prototipo y sus componentes, mientras que la Sección IV describe las situaciones de funcionamiento normal y de excepción contempladas por el sistema. Finalmente, la Secci ón $\mathrm{V}$ presenta las conclusiones y trabajos a futuro.

\section{DISEÑO DEL SISTEMA SAE}

El diseño del estacionamiento fue construido en base a un relevamiento de una muestra representativa de estacionamientos de la ciudad, lo cual permitió definir qué 
aspectos considerar en el prototipo para cubrir las necesidades de automatización real y de interés para la empresa solicitante, incluyendo la fácil adaptabilidad a variantes. El estacionamiento tiene una vía de ingreso y otra de egreso independientes. Ambas vías cuentan con una barrera vehicular, un detector magnético de presencia y dos cámaras IP. Además, en el ingreso se encuentra un mecanismo de detección de tipo o tamaño implementado con tres barreras infrarrojas que funcionan en conjunto con el detector magnético. La vía de entrada cuenta también con una pantalla que indica a los clientes el lugar asignado y otra información de interés, y una impresora de tickets. La Fig. 1 muestra un croquis de los periféricos principales en la vía de ingreso y egreso.
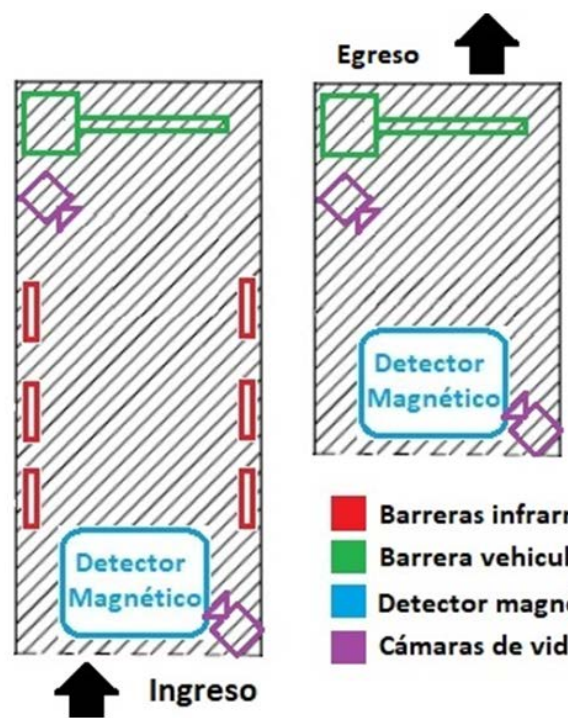

Barreras infrarrojas

Barrera vehicular

Detector magnético

Cámaras de video

Fig. 1. Croquis orientativo de la ubicación de los periféricos principales en el ingreso y la salida del estacionamiento.

La Unidad Central de Control (UCC) se encarga de interactuar con todos los componentes del sistema y procesar los datos obtenidos por los mismos. Entre las tareas asignadas, obtiene el número de patente del vehículo que ingresa o egresa y gestiona la base de datos local donde se guarda la información y características de cada vehículo estacionado. La Placa Principal del Sistema (PPS) concentra todos los periféricos de la zona de ingreso/egreso, menos las cámaras, y se comunica con la UCC mediante WiFi.

En cuanto al funcionamiento, el sistema detecta un vehículo que desea ingresar mediante un detector magnético adquirido ubicado en el suelo bajo el pavimento. Esto activa la obtención de la patente a través del análisis de video de las cámaras IP. La patente es buscada en la base de datos para ver si el cliente es abonado y, en caso de no ser encontrada se crea un perfil para el vehículo. Al mismo tiempo se determina el tamaño del vehículo y se lo añade al perfil, con el objetivo de determinar la tarifa a cobrar, generándose el ticket correspondiente. Una vez que el usuario retira el ticket se levanta la barrera y se permite el acceso. Si el usuario es abonado, no se entrega el ticket y se levanta la barrera. En forma similar al ingreso, el sistema de egreso comienza con la detección de un vehículo que desea retirarse, luego de lo cual se obtiene la patente con el fin de determinar si la tarifa ya fue pagada consultando a la base de datos. Realizado el pago, se levanta la barrera y se permite el egreso. Todo esto realizado en forma automatizada salvo el cobro del dinero que lo recibe un operario e indica en la base de datos a través de una interfaz del sistema, que ya fue efectuado el pago para permitir el egreso. En las Fig. 2 y Fig. 3 se pueden observar los diagramas de flujo correspondientes a la entrada y la salida de vehículos respectivamente.

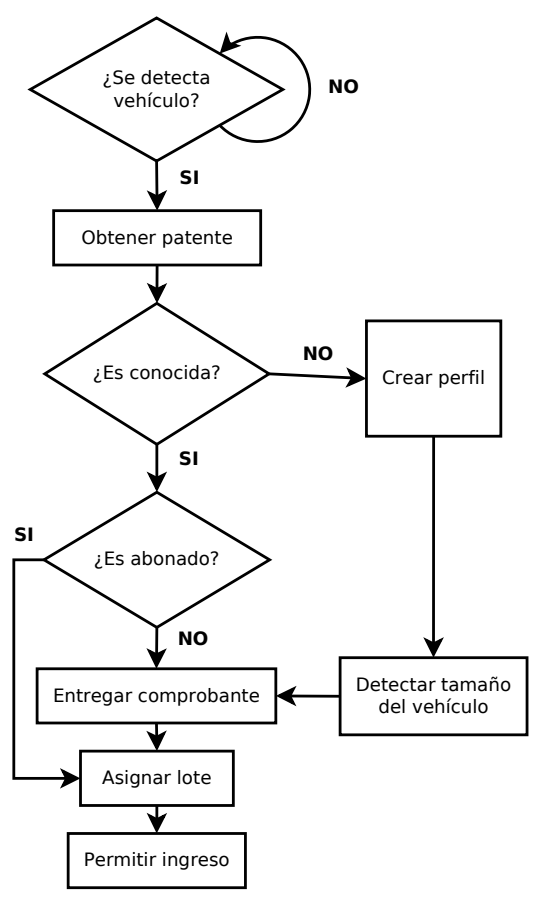

Fig. 2. Funcionamiento del sistema de estacionamiento en el ingreso.

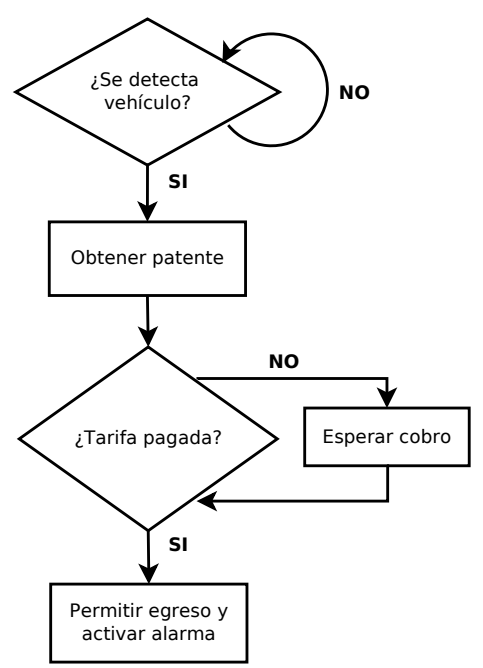

Fig. 3. Funcionamiento del sistema de estacionamiento en el egreso.

\section{CONSTRUCCIÓN DEL PROTOTIPO}

En el desarrollo del prototipo, el reconocimiento de la patente tanto del vehículo que ingresa como del que egresa es una de las etapas fundamentales, junto con el diseño y construcción de la PPS encargada de controlar los 
periféricos. A continuación se describen los componentes del sistema.

\section{A. Reconocimiento Automático de Patentes ALPR}

El reconocimiento automático de patentes (ALPR, del inglés Automatic License Plate Recognition) [3] es un problema típico que involucra varias ramas de estudio, principalmente al área de reconocimiento de patrones y el campo de la visión artificial, y que ya ha sido estudiado ampliamente [4] - [7].

Una de las principales dificultades consiste en que los escenarios pueden ser cambiantes, como podría ocurrir en el caso de un Sistema de Transporte Inteligente (ITS, del inglés Intelligent Transport System), donde el reconocimiento de patentes permite identificar vehículos en movimiento y obtener varias matriculas simultáneamente [7]. En nuestro caso, a pesar de que se considera que el sistema puede aplicarse a diversos tipos de estacionamiento, el escenario es más acotado: la cámara se encuentra en una posición fija y el vehículo frenando a baja velocidad. En este contexto, se tienen en cuenta otras posibles problemáticas, como las variaciones lumínicas (día/noche), la iluminación propia de la placa y la existencia de distintos modelos de placas patente en nuestro país: antiguo (1995 - 2016) y Mercosur (2016 - presente). Además, se consideran diferentes tipos de vehículo: automóviles, camionetas y motocicletas. La Fig. 4 muestra ejemplos de los formatos de patente considerados.
MERCOSUR
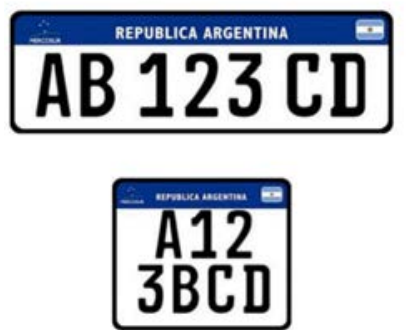

Antiguo
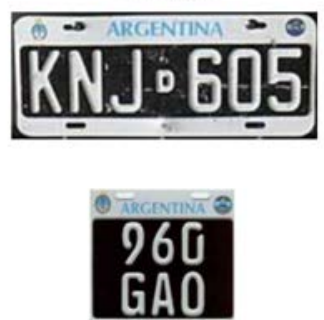

Fig. 4. Modelos de patentes argentinas vigentes: Mercosur y antiguo, en formato para automóvil/camioneta (primera fila) y motocicleta (segunda fila).

El sistema ALPR implementado se construyó en base al software libre de código abierto OpenALPR [8] bajo Linux. Este software utiliza el motor de OCR Tesseract [9]. La elección fue hecha luego de comparar OpenALPR con el sistema OpenCV 3 License Plate Recognition [10], también gratuito de código abierto y ampliamente difundido. Se observó que el primero se ajustaba más a las necesidades del SAE y permitía obtener resultados competitivos, mientras que el segundo no brindaba la misma disponibilidad de parámetros para el ajuste ni un motor de OCR tan potente. La adaptación de un sistema ALPR para modelos de patentes que no vienen incorporadas en el sistema no es una tarea trivial, ya que se requiere la modificación y ajuste de una gran cantidad de parámetros. Para adaptar el sistema OpenALPR a las patentes argentinas, se crearon y modificaron diferentes archivos, entre los que se mencionan:

- de configuración, con las medidas físicas de las placas patente, tamaño de los caracteres, cantidad máxima y mínima de caracteres por cada patente (7 para Mercosur y 6 para modelo antiguo), lectura de caracteres negros sobre fondo blanco y a la inversa, entre otros.

- de post-procesamiento, para validar la combinación de caracteres y números, lo cual elimina casos típicos de confusión como pueden ser la "O" y el "0", la "I" y el "1".

- general del sistema, permite mejorar el rendimiento. A modo de ejemplo, mencionamos: el parámetro detection_iteration_increase representa el porcentaje de incremento del cuadro del algoritmo LBP [11] (utilizado en la etapa de preprocesamiento) para cada iteración, donde cuanto más bajo es su valor, más lento es el sistema. Se modificó el valor por defecto de 1.1 a 1.7. El parámetro detection_mask_image permite definir una máscara que indica qué área de la imagen analizar en búsqueda de la patente. Por defecto se analiza toda la imagen y se modificó para que se tuviera en cuenta sólo la mitad inferior donde se espera encontrar la placa, reduciendo el tiempo total de procesamiento en aproximadamente $30 \%$.

- archivos alprd: vinculados con el procesamiento de video en tiempo real. Se definió una cola de trabajo para cada cámara, de forma de realizar el tratamiento de las patentes de los vehículos detectados tanto en la entrada como en la salida del estacionamiento en forma concurrente.

Cabe destacar que para el tratamiento de video en tiempo real, el sistema cuenta con un modo de funcionamiento denominado alprd (alpr daemon) el cual funciona en segundo plano y permite entregarle al sistema un stream de vídeo. Cuando el sistema detecta una matrícula en el vídeo, procesa el frame. A partir del mismo se obtiene el número de la patente junto con su medida de confianza, y algunas características adicionales como el tiempo de procesamiento y la ubicación de la patente en la imagen.

El sistema se ajustó para el reconocimiento de patentes argentinas vigentes en los formatos antiguo y Mercosur [12] para distintos tipos de vehículo como camionetas, autos y motocicletas. Para esto, se construyó un conjunto inicial de imágenes para el ajuste de los parámetros del sistema, y otro conjunto para el testeo con 330 muestras (165 para Mercosur y 165 formato antiguo) representativas para nuestro problema, incluyendo diferentes escenarios, condiciones lumínicas y desplazamientos de la patente con respecto a la cámara. En la Fig. 5 se pueden observar algunas imágenes del conjunto de prueba.

Los resultados fueron satisfactorios, logrando tasas de reconocimiento cercanas al $96 \%$ para el caso de vehículos con patentes argentinas del Mercosur y porcentajes cercanos al $94 \%$ para el formato antiguo. La Tabla I muestra los resultados para el conjunto de prueba.

TABLA I

PORCENTAJES DE PATENTES RECONOCIDAS CORRECTAMENTE PARA EL CONJUNTO DE PRUEBA CON 330 MUESTRAS.

\begin{tabular}{|c|c|}
\hline Modelo & $\boldsymbol{\%}$ \\
\hline MERCOSUR & 95.76 \\
\hline Antiguas & 93.94 \\
\hline
\end{tabular}



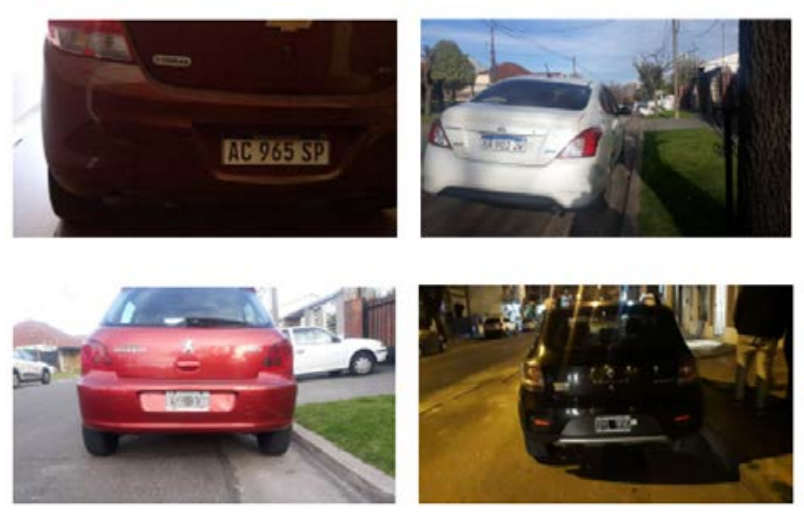

Fig. 5. Ejemplos de imágenes del conjunto de prueba para patentes de Mercosur (fila superior) y antiguas (fila inferior) tomadas en distintas condiciones de luminosidad y de ubicación del vehículo con respecto a la cámara.

\section{B. Placa Principal del Sistema}

Se diseñó y construyó la PPS encargada de controlar los equipos que se ubican en las zonas de ingreso y egreso. Los componentes principales que la integran son el microcontrolador ATmega328P y el módulo de comunicación WiFi ESP-01 que junto con un router permiten la comunicación con la UCC en la cabina a pocos metros de distancia (de ser necesario, podría incorporarse un repetidor de señal).

La inteligencia de la placa se encuentra en el microcontrolador, que se ocupa del sensado del estado de los periféricos y la distribución de las tareas a realizar, el control del encendido del módulo WiFi y la muestra de señales luminosas de alarma en caso de fallas. Además, se le brindó conectividad WiFi utilizando el módulo ESP-01. Estos dos se comunican entre sí mediante puerto serie.

El microcontrolador es de 8 bits y es fabricado por la empresa Atmel [13]. Cuenta con 28 pines, de los cuales 23 son líneas de entrada-salida programables. De ellas, 14 son digitales, de las que dos pertenecen a un puerto serial USART, utilizado para la transmisión de datos con el módulo de comunicación WiFi.

El módulo utilizado es el ESP-01, que es fabricado por AiThinker y se basa en la familia de módulos WiFi "ESP8266" de Espressif Systems. Este pequeño módulo puede utilizarse como adaptador Wi-Fi, que es la función que cumple en este proyecto. Finalmente, el mismo posee ocho pines. Entre estos se encuentran un reset, un pin de habilitación, dos pines de alimentación y cuatro pines digitales, correspondiendo dos de ellos a una UART. Esta se utiliza para la comunicación con el microcontrolador, mientras que uno de los pines digitales permite implementar una señal luminosa en caso de falla. Ambos componentes se observan en la Fig. 6.

En el proyecto se contempló el diseño y construcción de una fuente de tensión regulada que se encuentra integrada en la PPS. Su propósito es alimentar la placa y los periféricos. Para ello, cuenta con salidas de $12 \mathrm{~V}$, $5 \mathrm{~V}$ y $3.3 \mathrm{~V}$ de tensión continua. Las mismas se encargan de alimentar los periféricos, el microcontrolador y el módulo WiFi, respectivamente. Asimismo, cuenta con una protección frente a sobretensiones en el lado primario de su transformador.

Respecto al conexionado de los periféricos, se debe con-
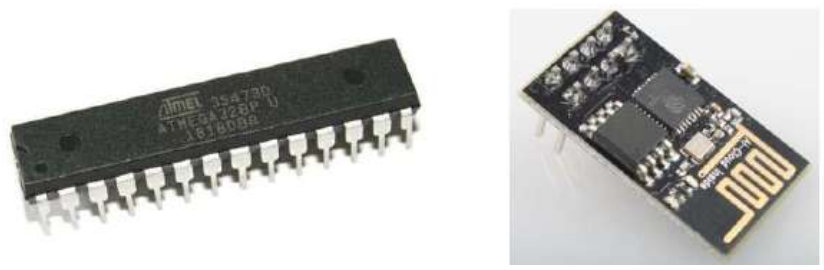

Fig. 6. Componentes principales de la PPS. A la izquierda, el microcontrolador ATmega328P. A la derecha, el módulo de comunicación WiFi ESP-01.

siderar que algunos de ellos han sido simulados debido a una cuestión de costos o a que han quedado fuera del alcance del proyecto. En cuanto a los que se encuentran simulados, como es el caso de las barreras vehiculares de entrada y salida, la expendedora de tickets del ingreso y el detector magnético de salida, la conexión con el microcontrolador es directa, mediante sus pines digitales de entrada. Mientras que la elevación de cada una de las barreras y la impresión del ticket se visualizan mediante un led, el segundo detector magnético y el retiro del ticket se simulan con pulsadores. Aquellos que se encuentran implementados, que son las barreras infrarrojas que forman parte del sistema de detección de tamaño y el sensor magnético de presencia del ingreso, se conectan con el microcontrolador a través de una adaptación de niveles de tensión, dado que el microcontrolador y los periféricos trabajan con diferentes niveles lógicos.

Adicionalmente, la PPS cuenta con leds indicadores que se encienden en caso de producirse alguna falla en el microcontrolador o el módulo de comunicación.

Finalmente, se realizó el diseño de la PPS en Altium Designer y se la construyó. Inicialmente, la misma fue implementada mediante un Arduino Mega. El uso de esta placa de desarrollo permitió ensayar el funcionamiento de los códigos que se fueron realizando al comienzo del proyecto. Posteriormente, la misma fue reemplazada por un circuito construido utilizando protoboards, implementado con el microcontrolador ATmega328P. Una vez que se verificó que el circuito desarrollado funcionaba en forma correcta, se procedió a realizar el diseño del PCB correspondiente. El resultado obtenido se observa en la Fig. 7.

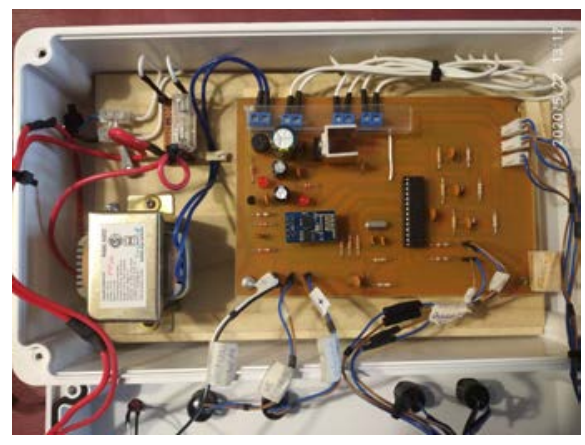

Fig. 7. PPS dentro de la caja del prototipo final.

\section{Periféricos}

Se implementó un sistema para determinar, en base a su longitud, la categoría del vehículo y la correspondiente tarifa. Consiste en un arreglo de 3 barreras infrarrojas 
dispuestas a 2.5 metros de distancia entre sí de forma tal de determinar la categoría en función de cuántas barreras son activadas por el vehículo cuando éste está frente a la barrera de ingreso. En base al orden en que las mismas son activadas con el paso del vehículo, por software se determina la categoría.

Las barreras utilizadas tienen un alcance $\leq 15 \mathrm{~m}$. Cada equipo transmisor y receptor permite ajustar la frecuencia de trabajo de la barrera y evitar interferencias con otras cercanas. Una de las barreras puede observarse en la Fig.8.

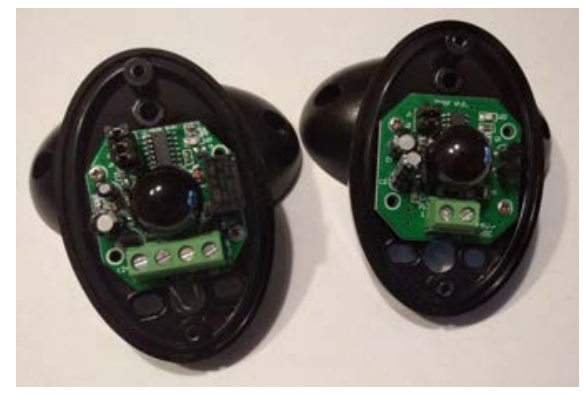

Fig. 8. Barrera infrarroja utilizada: receptor (izquierda) y transmisor (derecha).

Por su parte, el detector de presencia magnético posee un lazo inductivo que debe realizarse con cable de 1.5 $\mathrm{mm}^{2}$ de área y debe tener forma rectangular. En base al manual de usuario y al tamaño de las vías de acceso de los establecimientos relevados, se consideró un lazo de $2.5 \mathrm{~m}$ de largo por $1 \mathrm{~m}$ de ancho, al que le corresponden cuatro vueltas de cable. El equipo posee dos indicadores leds, uno rojo y uno verde. Mientras el equipo se encuentre alimentado, el primero estará siempre prendido. El verde va a parpadear en caso de falla o va a quedar encendido cuando se detecte un vehículo atravesando el lazo. El detector de presencia implementado se ve en la Fig. 9.

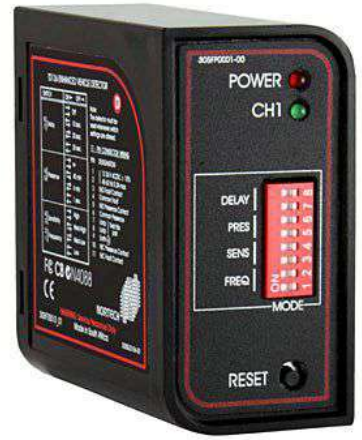

Fig. 9. Detector magnético utilizado.

\section{Unidad Central de Control y Base de Datos}

La UCC se implementó en una PC y es la encargada del procesamiento de las imágenes, el reconocimiento de matrículas y el almacenamiento de los datos de los clientes. En ella se ejecuta el módulo principal del sistema encargado de la sincronización de procesos. Cada cámara captura video en forma permanente y está asociada a una cola de trabajos que contiene las patentes reconocidas en tiempo real. La
UCC se encarga de determinar qué patentes analizar cuando el detector magnético indica la presencia de un vehículo.

El uso de un sistema que capture los datos de la matrícula de un vehículo y lo almacene en una base de datos en formato de texto evita la necesidad de ocupar espacio de memoria debido al almacenamiento de videos o imágenes y facilita la consulta de los datos de cada uno de los clientes del establecimiento, como así también permite la sincronización de los procesos por parte del sistema desde la UCC.

La base de datos lleva registro de los clientes abonados que pagan mensualmente, los vehículos estacionados en un momento dado, y el histórico de las estadías. Para la implementación se utilizó el sistema de gestión de bases de datos MySQL [14]. En la tabla de los vehículos estacionados "Parking" se almacena el número de patente, si el cliente es abonado o no, el tipo de vehículo, el pago efectuado y la fecha y hora de entrada y salida. En la tabla "History" se mantienen los datos de los vehículos que ya abandonaron el estacionamiento.

Cuando el detector magnético detecta la presencia de un vehículo en la vía de egreso, avisa a la UCC para que verifique el registro del pago en la base de datos y de esta manera levante la barrera de salida. Luego de esto, se agrega en la tabla History la estadía del vehículo y se elimina de la tabla Parking. Las Fig. 10.a y Fig. 10.b muestran una instancia para la tabla de vehículos estacionados y el histórico respectivamente. Se observa que el auto con patente KNJ605 efectuó el pago de la estadía y quedó registrado en la tabla Parking. Este hecho indica que el vehículo aún no egresó del estacionamiento. La tabla History de la Fig. 10.b muestra, en un momento posterior, el registro de la estadía de dicho vehículo ya en el histórico indicando que el mismo ya egresó y fue eliminado de la tabla Parking. Cabe señalar que en esta versión del sistema y a difencia del resto de las acciones, el pago de la estadía no está automatizado y es gestionado y registrado en la base de datos por un operador humano, a través de una interfaz.

\section{E. Sincronización de procesos}

El prototipo final se completa con la sincronización de procesos que puedan ocurrir en simultáneo como lo es el ingreso y egreso de vehículos. Esto se implementó en el framework YAKINDU Statechart Tools [15] bajo licencia académica, utilizando máquinas de estado conformadas por diagramas UML y desarrollando los códigos en lenguaje $\mathrm{C}++$ distribuidos en la UCC, PPS y módulo WiFi (en este último modificando firmware original y uso de libreria pública [16]). Esto ha posibilitado el entrelazamiento de operaciones para implementar el sistema en base a la técnica de multiprogramación, de forma tal que el procesador ejecute las tareas en forma concurrente.

La fase de conexión UCC-PPS-WiFi inicia el sistema quedando luego listo para operar en forma continua. En caso de fallo, el sistema informa por pantalla.

En la Fig. 11 se puede observar el diagrama de bloques representativo de la comunicación dentro del sistema. Los tres subsistemas UCC, WiFi y PPS se comunican a través de mensajes, cada uno de los cuales es contestado por el receptor con un ACK. El envío y recepción de mensajes es 


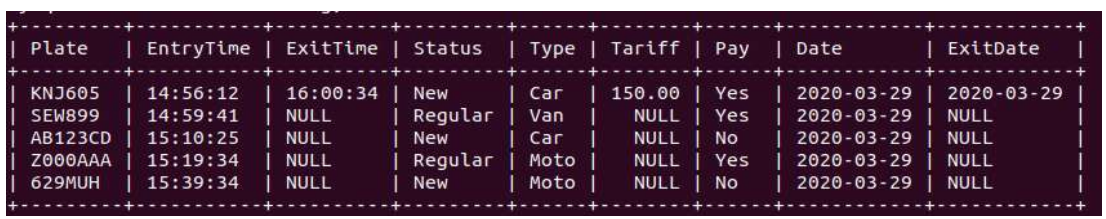

(a) Tabla Parking

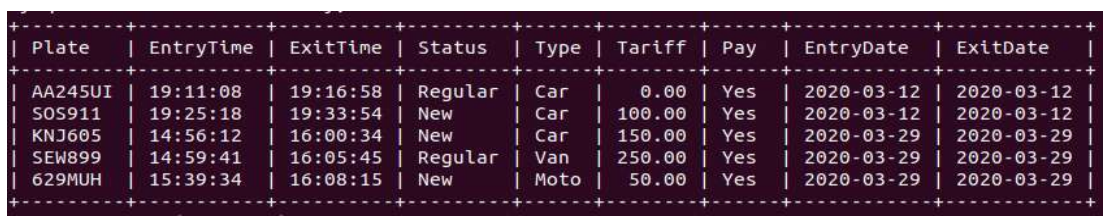

(b) Tabla History

Fig. 10. Instancia de ejemplo para las tablas de la base de datos que registran los vehículos estacionados y el histórico.

la función principal del módulo WiFi, intermediario entre la PPS y la UCC.

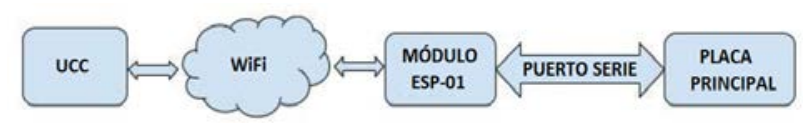

Fig. 11. Comunicación dentro del sistema entre la UCC, módulo ESP-01 y la PPS.

Tanto la UCC como la PPS disponen de una región destinada a las tareas que deben llevar a cabo cuando se acciona la via de ingreso o egreso. A modo de ejemplo, la Fig. 12 muestra el procedimiento sobre la vía de ingreso de la PPS.

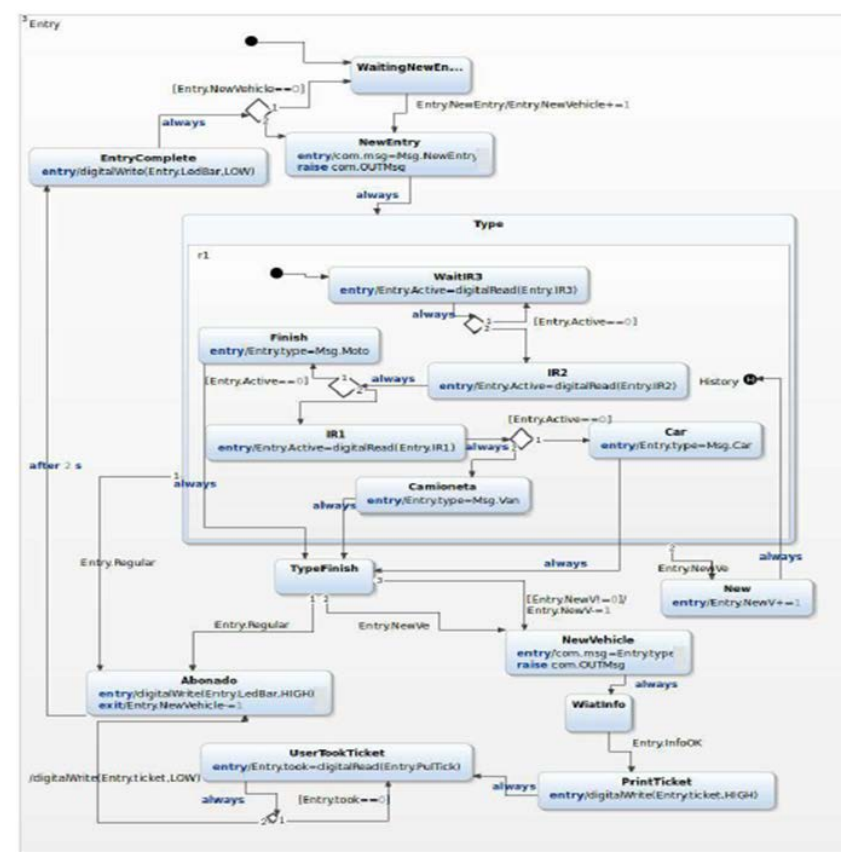

Fig. 12. Procedimiento de la PPS sobre la vía de ingreso.

\section{PRuebas de funcionamiento y MANEJo DE EXCEPCIONES}

El sistema contempla situaciones de excepción, como por ejemplo la asignación de un identificador para patentes dañadas, lectura no concordante de la misma patente en el ingreso y egreso, o que no han podido ser leídas correctamente. Adicionalmente, la placa principal cuenta con leds indicadores en caso de fallas en el microcontrolador o el módulo WiFi, y mensajes de error que la Unidad Central presenta en pantalla en estas oportunidades.

En cuanto al ingreso, si al realizar la detección de la patente el software no logra obtenerla, ya sea porque el vehículo no la posee, o bien porque está muy dañada, el sistema le otorga un identificador que consta de siete números. En estos casos, el vehículo es considerado como un nuevo cliente, por lo que el mecanismo de clasificación de vehículos debe determinar su tamaño antes de que este ingrese.

Respecto al egreso, pueden darse varias situaciones. $\mathrm{Si}$ un vehículo se encuentra sobre la vía de salida, pero en la base de datos no figura que haya realizado el pago de la tarifa, el sistema se mantiene continuamente comprobando el estado del pago. Cuando el mismo es efectuado, se permite la salida. Por otra parte, si el cliente, sin haber abonado, se retira de la vía de egreso hacia el interior del establecimiento, la PPS le informa esto a la UCC para que vuelva a esperar un nuevo vehículo. Como en la salida también se realiza la detección de la matrícula, puede ocurrir que la misma no sea encontrada en la base de datos. Entonces, el sistema le pregunta al operario si se trata de un error de lectura o de algún caso particular. En el primer caso, la patente podría haber sido mal reconocida a la entrada o la salida, o bien tener asignada una patente provisoria. Por lo tanto, el operario debe ingresar la patente que figura en el ticket que se le entregó al cliente en la entrada, con el cual realizó el pago. Una vez ingresada, la UCC busca esta nueva patente y procede en forma normal. Si se trata de un caso particular, el sistema le niega la salida al cliente y el operario debe pedirle al mismo que retire el vehículo de la vía de egreso para aclarar su situación.

Por otra parte, anteriormente se mencionó que la placa cuenta con leds indicadores en caso de fallas de hardware. Uno de ellos se utiliza para señalar que el módulo ESP-01 no logra conectarse a la red WiFi generada por el router en el lapso de 10 segundos programado. En ese caso, el led empezará a parpadear. Sin embargo, esto no afecta al funcionamiento del microcontrolador, el cual presentará en pantalla el mensaje "Fallo de conexión". También se cuenta con un led destinado a indicar la existencia de fallas en el 
microcontrolador. Luego de la inicialización del sistema, el mismo parpadeará en caso de que se produzca un error en la comunicación entre la placa y el módulo WiFi, debido a la pérdida de mensajes entre ellos.

Por último, cabe señalar que para la construcción del prototipo se realizaron pruebas en laboratorio y en un primer escenario externo con características similares a un estacionamiento considerado estándar según el relevamiento realizado. Para el siguiente prototipo se planean realizar ensayos funcionales en ambientes similares al de uso final.

\section{Conclusiones y Trabajos Futuros}

Se ha presentado un prototipo para un sistema automatizado de estacionamientos para patentes argentinas desarrollado para una empresa de la ciudad de Mar del Plata, que ha funcionado satisfactoriamente. Este desarrollo surge de la necesidad de automatizar los estacionamientos a nivel local frente al crecimiento del parque automotor. El prototipo es fácilmente adaptable a variantes, como en la disposición de la entrada y salida, y permite bajar costos de desarrollo y mantenimiento.

El sistema implementa un sistema de reconocimiento automático de patentes argentinas con el que se han obtenido porcentajes de reconocimiento similares a los encontrados en la documentación analizada. Posee además un mecanismo de detección de tamaño estandarizado, que se basa en el largo del vehículo, en la vía de entrada (los establecimientos relevados dejaban esta diferenciación a criterio del operario). De esta forma el cálculo de la tarifa a cobrar es automático. Adicionalmente, implementa una base de datos que registra los vehículos que se encuentran en el establecimiento, los clientes abonados y mantiene un historial de las estadías. Toda esta información es relevante para la gestión de los estacionamientos y para datos estadísticos, entre otras aplicaciones.

El prototipo considera una vía de ingreso y otra de egreso permitiendo la entrada y la salida de vehículos en simultáneo, lo cual es manejado por el sistema.

A futuro, se implementarán técnicas para seguridad en las comunicaciones, manejo automatizado de fallos de dispositivos y se sumará la automatización total del cobro de la estadía y la administración inteligente del espacio.

\section{REFERENCIAS}

[1] M. Osorio. (2018) La flota circulante de Argentina creció $30 \%$ en 6 años. [Online]. Available: http://www.carsdrive.com.ar/noticias/novedades/la-flotacirculante-de-argentina-crecio-30-en-6-anos/

[2] KinouWell. Off street parking solution. [Online]. Available: https://www.kinouwellparking.com/solutions/off-street-parkingsystem/

[3] A. Agbemenu, J. Yankey, and E. O., "An automatic Number Plate Recognition System using OpenCV and Tesseract OCR Engine," International Journal of Computer Applications, vol. 180, pp. 1-5, May 2018.

[4] I. Moretti, J. Jorge, J. Amado, C. Caniglia, and D. Puntillo, "Software libre para reconocimiento automático de las nuevas patentes del Mercosur," 2016, Instituto Nacional de Tecnología Industrial (INTI). Córdoba, Argentina. Premiado en el XVII Congreso argentino de Vialidad y Tránsito realizado en el año 2016 como el mejor trabajo del área de tecnología inteligente.

[5] R. E. Sorace, V. S. Reinhardt, and S. A. Vaughn, "High-speed digitalto-RF converter," U.S. Patent 5668842 , Sep. 16, 1997.
[6] S. Du, M. Ibrahim, M. Shehata, and W. Badawy, "Automatic License Plate Recognition (ALPR): A State-of-the-Art Review," IEEE Transactions on Circuits and Systems for Video Technology, vol. 23, pp. 311-325, Feb. 2013, doi: 10.1109/TCSVT.2012.2203741.

[7] C. Patel, D. Shah, and A. Patel, "Automatic Number Plate Recognition System (ANPR): A survey," International Journal of Computer Applications (IJCA), vol. 69, May 2013, doi: 10.5120/11871-7665.

[8] P. Recognizer. Accurate, Fast, Developer-Friendly ANPR. [Online]. Available: https://platerecognizer.com/

[9] R. Smith, "An Overview of the Tesseract OCR Engine," in Proc. Ninth Int. Conference on Document Analysis and Recognition (ICDAR), 2007, pp. 629-633.

[10] C. Dhams. OpenCV $3 \mathrm{KNN}$ Character Recognition. [Online]. Available: https://tinyurl.com/tgzd2ww

[11] LBP and ULBP - Local Binary Patterns and Uniform Local Binary Patterns. [Online]. Available: https://tinyurl.com/txfflkq

[12] C. del Mercado Común (CMC), "Reglamentación Patente Única del MERCOSUR,” pp. 3-4, ago 2014.

[13] ATmega328P. able: https://www.alldatasheet.com/datasheetpdf/pdf/313656/ATMEL/ATmega328P.html

[14] Oracle MySQL: The Most Popular Open Source Database. [Online]. Available: http://www.oracle.com/us/products/mysql/overview/index.html

[15] YAKINDU Statechart Tools. Use the power of state machines. [Online]. Available: https://www.itemis.com/en/yakindu/state-machine/

[16] ESP8266WiFi.h Arduino free library. [Online]. Available: https://tinyurl.com/y42dzllp 